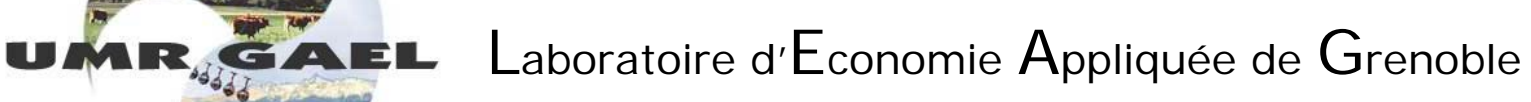

CLICK'N'ROLL: NO EVIDENCE OF ILLUSION OF CONTROL

FILIPPIN Antonio ; CROSETTO Paolo

- April 2015 -

JEL CODES : B49 ; C91 ; D81

Working Paper GAEL ; 2015-06 


\title{
Click'n'Roll: No Evidence of Illusion of Control th
}

\author{
Antonio Filippin ${ }^{\mathrm{b}, \mathrm{c}}$, Paolo Crosetto ${ }^{\mathrm{a}}$ \\ a INRA, UMR 1215 GAEL, Univ. Grenoble Alpes, 38000 Grenoble, France. \\ ${ }^{b}$ University of Milan, Department of Economics, Via Conservatorio 7, 20122 Milano, Italy \\ 'Institute for the Study of Labor (IZA), Schaumburg-Lippe-Str. 5-9, 53113 Bonn, Germany
}

\begin{abstract}
Evidence of Illusion of Control - the fact that people believe to have control over pure chance events - is a recurrent finding in experimental psychology. Results in economics find instead little to no support. In this paper we test whether this dissonant result across disciplines is due to the fact that economists have implemented only one form of illusory control. We identify and separately tests in an incentive-compatible design two types of control: a) over the resolution of uncertainty, as usually done in the economics literature, and b) over the choice of the lottery, as sometimes done in the psychology literature but without monetary payoffs. Results show no evidence of illusion of control, neither on choices nor on beliefs about the likelihood of winning, thus supporting the hypotheses that incentives crowd out illusion of control.
\end{abstract}

\section{JEL Classifications: B49; C91; D81}

Keywords: Illusion of Control, Experiment, Risk Elicitation, Hypothetical Bias

\section{Introduction}

Langer (1975) defines illusion of control as an expectancy of a personal success probability inappropriately higher than the objective probability would warrant. ${ }^{1}$ Skills and luck contribute jointly to determine outcomes in most real life situations. Skills play nonetheless no role in many situations in which the outcome is driven by uncontrollable random events. Illusion of control induces subjects to perceive a positive degree of control in pure chance events, and the straightforward consequence is a more risk seeking behavior.

The psychology literature finds robust effects of illusion of control under a variety of circumstances and designs, as shown for instance in the meta-analysis by Presson and Benassi (1996). The degree of expertise (Langer, 1975) or familiarity (Benassi et al., 1979) with the

\footnotetext{
${ }^{\sqrt{3}}$ We are grateful to the Max Planck Institute in Jena for financial and logistic support and to Florian Strum and Nadine Marmai for excellent lab assistance. We thank the participants to the ESA European meeting in Prague for insightful comments. All remaining errors are ours.

Contact: antonio.filippin@unimi.it (Antonio Filippin), paolo.crosetto@gmail .com (Paolo Crosetto)

${ }^{1}$ Although implicitly assuming the existence of an objective measure of the probability of success that is often not available (Presson and Benassi, 1996), this definition is still useful for illustrative purposes. Moreover, experimental evidence is usually obtained manipulating the intensity of the treatment aimed at triggering illusion of control. Therefore, causal evidence of the (qualitative effects of) illusion of control can be identified even when lacking any objective probability of success.
} 
randomization devices have been shown to affect the degree of illusory control. Subjects think that the chance of success is higher when they are more involved in the randomization procedure, as when they can choose the target numbers in a lottery game (Dunn and Wilson, 1990; Langer, 1975) or when the outcome of the lottery is determined rolling a die directly instead of having the experimenter doing so (Dunn and Wilson, 1990). When skills are actually important, letting subjects be more involved, train, and familiarize with the task increases their likelihood of succeeding. The inference humans make from real life that skills make a difference appears to carry over to situations in pure chance events, such as lotteries, where the subject has no control.

Different results have been found in the economics literature. Economists have focused mainly on choices under risk, finding invariably little or no evidence of illusion of control. For instance, Charness and Gneezy (2010) implement an Investment Game (Gneezy and Potters, 1997) in which subjects can choose 3 'winning' numbers out of 6 . They find that a majority of subjects (68\%) prefers to roll the die if this is costless, but only a small minority $(9 \%)$ does so when rolling the die costs $5 \%$ of their endowment. Similar results have been found by Li (2011), who finds that subjects may even display different preferences, including preferences for no control (paying to be released from having to choose the numbers), and preferences for randomization (paying to let a coin toss decide who will pick the numbers). ${ }^{2}$ Poon (2011) also finds that most of the subjects display indifference in a multiple price list task aimed at eliciting their willingness to pay up to one dollar to exercise control in a lottery.

One possible explanation for this striking difference in findings might be given by hypothetical bias: illusion of control would be observed in hypothetical choices, but be crowded out by monetary incentives. Interestingly, Dunn and Wilson (1990) report that illusion of control is reduced even when inflating hypothetical payoffs in a non-incentivized experiment.

Another possible reason for the dissonance in results is that the literature in experimental economics has been restricted to one type only of illusion of control, obtained by giving subjects (illusory) control over the resolution of uncertainty. This has usually been done by letting subjects roll the dice, or asking their willingness to pay to have control over the rolling. There is, however, another form of illusory control over more concrete aspects of the choice itself. In the psychological literature this has been done, e.g., by allowing subjects to choose and trade their lottery ticket rather than being assigned one (Langer, 1975), or by asking subjects to press buttons that might or might not have consequences (Gino et al., 2011). In other words, a greater involvement can concern the choice of which lottery should be played, instead of how to resolve uncertainty of a given lottery (who should roll the die, which are the outcome numbers, etc.). These two phases of a decision under risk are clearly distinct from a theoretical point of view, thereby identifying two different kinds of illusion of control, which do not necessarily have similar effects.

Moreover, real life decisions under risk can usually be described as the choice of a lottery: imagine an investor who chooses between assets characterized by different risk profiles, a citizen who fills the tax declaration, a patient that follows a therapy, the decision to subscribe an insurance contract as well as the possible moral hazard problem that follows, and so on.

\footnotetext{
${ }^{2} \mathrm{Li}$ (2011) attributes his results to preferences for different sources of uncertainty (Chew and Sagi, 2008; Tversky and Wakker, 1995) rather than to illusion of control.
} 
In all these circumstances, the choices made affect the nature (payoffs and probabilities) of the lottery that will be played, while they do not usually affect the decision over the final outcome. It is indeed very difficult to imagine a situation in which the agents are given active involvement in the resolution of uncertainty, as even at the casino players are not given the option to spin the roulette by themselves. In contrast, the experimental evidence on illusion of control in economics has been gathered focusing on the resolution of uncertainty only.

In this paper we test whether the striking difference of results across disciplines is due to the fact that experimental economists have restricted their attention to one and not all possible forms of illusory control. We do so setting up a design that clearly identifies and separately tests in an incentivized setting the illusion of control over the choice (or definition) of the lottery vs. the illusion of control over the resolution of uncertainty. ${ }^{3}$

Towards this goal we exploit some useful features of the Bomb Risk Elicitation Task (BRET) (Crosetto and Filippin, 2013). The BRET can be manipulated to incorporate both types of illusion of control in a homogeneous environment, thereby making the results comparable. The BRET allows us on the one hand to replicate the manipulations traditionally employed in the economics literature, by letting the subjects roll the dice that determine the position of the bomb. On the other hand, it allows us to vary the degree of control in the collection process itself, by allowing subjects to click to collect every single box vs. just choosing a stopping point. Note that in the BRET the choice made by the subjects determines simultaneously both the amounts at stake and the probability of securing them, making the BRET rather similar to most of the aforementioned real life situations, in which the choices affect directly outcomes and probabilities.

We find null results across the board. Neither the perceived probability of winning nor the choice made react to the experimental manipulations. While increased involvement in the resolution of uncertainty was already known to have little to no effect when incentives matter, our experiment sheds light on the fact that null results extend to an increased involvement of the subjects in the generation of the lottery. According to our results, the disappearance of the illusion of control finding in economics experiments as compared with the psychological literature is due to the role of incentives alone.

The paper is organized as follows: in Section 2 and 3 we illustrate the design and procedure of our experiment. Section 4 presents our findings, while Section 5 summarizes and concludes.

\section{Experimental Design}

Our treatments are all built on the Bomb Risk Elicitation Task (BRET), a visual real time risk elicitation task introduced by Crosetto and Filippin (2013). Subjects face a $10 \times 10$ square in which each numbered cell represents a box. They are told that 99 boxes are empty, while

\footnotetext{
${ }^{3}$ Note that distinguishing the two phases of decision under risk also has the advantage of allowing to better rationalize previous findings. For instance, Charness and Gneezy (2010) report that the amount invested does not differ, regardless of subjects expressing preferences for control or not. Similarly, Li (2011) finds that less than $5 \%$ of the subjects believe that having control will increase their probability of winning, even among those who choose to have control. In other words, even when subjects express a preference for control over the resolution of uncertainty (likely because it is costless), their behavior in the choice of the lottery does not significantly differ.
} 
one contains a time bomb programmed to explode at the end of the task, i.e., after choices have been made. The position of the time bomb $b \in[1,100]$ is randomly determined after the choice is made with a roll of two 10-sided dice, allowing to generate random uniform numbers from 1 to 100 . Calling $k$ the number of boxes collected, if $k_{i}^{*} \geq b$ it means that subject $i$ collected the bomb, which by exploding wipes out the subject's earnings. In contrast, if $k_{i}^{*}<b$, subject $i$ leaves the minefield without the bomb and receives 10 euro cents for every box collected. The metaphor of the time bomb allows to implement a choice in strategy method, avoiding the truncation of the data that would happen in case of a real-time notification.

Subjects' decision can be formalized as the choice of their favorite among the set $\mathcal{L}$ of 101 lotteries fully described both in terms of probabilities and outcomes by the stopping point $k \in[0,100]$,

$$
\mathcal{L}=\left\{\begin{array}{ll}
0 & \frac{k}{100} \\
0.1 k & \frac{100-k}{100}
\end{array} .\right.
$$

$k$ drives at the same time the change of probabilities and the amounts of money at stake, summarizing the trade-off between the amount of money that can be earned and the likelihood of obtaining it. The degree of risk aversion negatively correlates with the choice of $k$ and a risk-neutral subject should choose $k=50$.

\subsection{Baseline Treatment: the classic Bomb Risk Elicitation Task}

In the Baseline condition there are a "Start" and a "Stop" button below the square. From the moment the subject presses "Start" one box is automatically collected per second, starting from the upper left corner of the square. A screenshot of the task after 35 seconds (i.e., after 35 boxes have been collected) as shown to the subjects is reported in Figure 1. The subject is informed about the number of boxes collected at any point in time. Each time a box is collected, the subject's provisional account is credited with 10 additional euro cents. ${ }^{4}$ The subject can, at any time, stop the drawing process by hitting the "Stop" button, thus determining the preferred number of boxes to collect, $k \in[0,100]$.

In the baseline treatment, subjects were exposed to a one-shot standard BRET, followed by a questionnaire. At the end of the experiment, the experimenters went around the lab to roll individually and privately in each cubicle the two ten-sided dice used to determine the position of the bomb.

\subsection{Control over the resolution of uncertainty: 'Roll'}

We introduce illusion of control over the resolution of uncertainty by allowing the subjects to roll the dice themselves. This minimal manipulation has been around at least since Dunn and Wilson (1990) and has been commonly used in experimental economics (Charness and Gneezy, 2010; Li, 2011). The subjects go through a standard BRET in all details identical to the baseline treatment, but they roll individually and privately the dice to determine the position of the bomb. The subjects are aware that they will be in charge of the rolling from the very beginning of the experiment.

\footnotetext{
${ }^{4}$ The expected value of of the BRET task for a risk neutral player is therefore 2.5 euro.
} 
Euro: 3.5

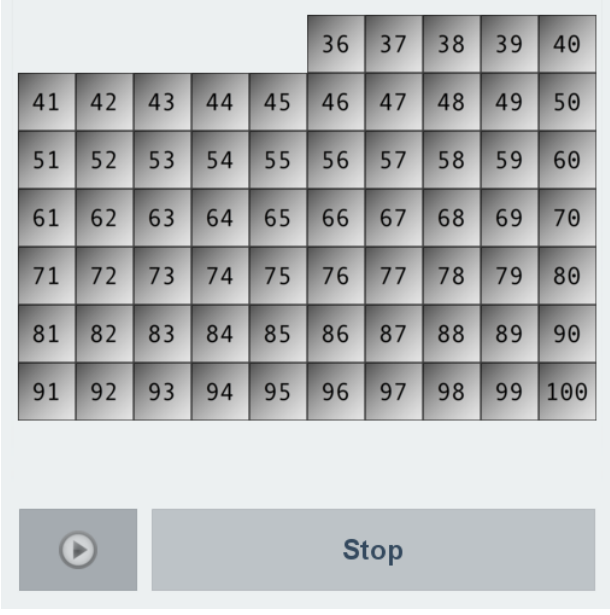

Number of boxes collected

35

Number of remaining boxes

65

Figure 1: The BRET interface after 35 seconds

We run two different treatments, increasing in the degree of familiarity with the dice. In the Roll treatment the subjects receive the dice at the end of the experiment, are allowed to roll twice for practice, and then roll once to determine the position of the bomb. In the Roll with Experience treatment, the subjects receive the dice at the beginning of the experiment, and are given extra time to familiarize with them before going through the BRET.

\subsection{Control over the choice of the lottery: 'Click'}

We introduce control over the shaping of the lottery by slightly modifying the BRET. In the Click treatment, we manipulate the way in which the subjects make their choice. Instead of stopping an automatic process like in Baseline, subjects are asked to click to collect every single box. In the BRET interface the "Start" button is replaced by a "Collect" button. Each time that the subjects press "Collect", one box is collected. The subjects have hence to click several times to reach their desired stopping point. This procedure leads to a more active involvement in the choice of the preferred lottery. In this treatment the resolution of uncertainty is identical to the Baseline condition, as the dice are rolled by the experimenters.

\section{Procedures}

The experiment was run in January 2013 at the laboratory of the Max Planck Institute of Economics in Jena, Germany. We implemented a pure between-subjects design. 86 subjects took part to the Baseline BRET treatment, 93 to Roll, 62 to Roll with Experience, and 90 to the Click treatment, for a total of 331 subjects distributed over 11 sessions, each lasting about half an hour. The sample includes mainly students from the Friedrich Schiller University Jena, Germany.

The experiment was computerized. The experimental software was programmed in Python (van Rossum, 1995). 
Upon entering the lab, subjects were randomly assigned to a computer. Subjects found noise-reducing acoustic earmuffs on their desk. We introduced earmuffs to avoid the strong herd and contagion effects that could otherwise occur in the Click treatment. In fact, Crosetto and Filippin (2015) show that subjects substantially change their choices when hearing each others' clicking. In order not to bias results, the earmuffs were used in all treatments alike.

Instructions were displayed on the screen and read aloud. ${ }^{5}$ Then subjects answered a set of multiple-choice control questions. No subject was allowed to continue until all control questions had been answered correctly. We recorded the number of errors submitted in the control questions and the time needed to clear the control question screen to be used as a control in the data analysis. After all subjects had correctly answered all control questions, and after the odd-out clarifying questions were individually addressed, subjects were asked to wear the earmuffs and went through a trial period in order to experience the visual representation of the task. At the end of the trial period, however, there was no draw of the bomb's position in order not to provide the subjects with reference points. The paying task was then played one-shot.

Subjects were then asked to remove the earmuffs and fill in a questionnaire containing demographic questions (age, gender, number of siblings, field of study), the SOEP selfreported risk question, ${ }^{6}$ a self-reported measure of difficulty with the task, on a Likert scale from 0 (easy) to 10 (hard) and a question asking them to report their beliefs on the probability of winning. The reason why we directly ask for the perceived probability of winning is that involvement in the resolution of uncertainty has been already shown to have little to no effect on choices in other economics experiments (Charness and Gneezy, 2010; Li, 2011). Therefore, a direct measure of illusion of control that elicits subjective probability of success is more likely to detect a possible weak effect of the treatment. ${ }^{7}$

At the end of the experiment the uncertainty surrounding the position of the bomb was resolved by means of the rolling of two ten-sided dice. The exact procedures for the rolling were treatment-dependent, as described above in Section 2.

\section{Results}

The average earning was 4.58 euro, including a show-up fee of 2.5 euro, in line with usual earnings for short experiments in Jena.

Table 1 reports descriptive statistics on the average choice in the task by treatment. Choices are rather similar across treatments and fairly close to risk neutrality.

\footnotetext{
${ }^{5}$ The English translation of the original German instructions is available in Appendix A.

${ }^{6}$ We employed the general risk question used in the German Socio-Economic Panel (SOEP, see Wagner et al., 2007) on a $0-10$ scale: "How do you see yourself: are you generally a person who is fully prepared to take risks or do you try to avoid taking risks?" The validity of this self-reported question to elicit risk attitudes as compared to the results of incentivized lottery-based tasks has been explored by Dohmen et al. (2011), who find that self-reported answers can represent a valid low-cost substitute for incentivized lottery schemes, although the fraction of variance explained is quite low (about $6 \%$ ).

${ }^{7}$ Presson and Benassi (1996) argue that the measured effects of illusion of control are lower when subjects are directly asked the probability of success, because this is likely to make them realize that indeed such a probability cannot have changed because of the increased involvement. Our design cannot trigger such an effect because the probability is asked after the choice has been made.
} 


\begin{tabular}{lcccc}
\hline & \multicolumn{2}{c}{ Mean choice } & \multicolumn{2}{c}{ SOEP } \\
& mean & st.dev. & mean & st.dev. \\
\hline BRET & 49.15 & 11.78 & 4.94 & 2.09 \\
Roll & 50.27 & 12.86 & 5.27 & 2.05 \\
Roll Experience & 49.21 & 10.28 & 5.18 & 1.85 \\
Click & 47.13 & 12.53 & 4.88 & 1.95 \\
\hline
\end{tabular}

Table 1: Mean choice and SOEP self-reported risk attitudes, by treatment

Only the Click treatment displays a slightly lower point estimate, i.e. in the opposite direction as the expected effect of illusion of control, but not significantly so. In fact, KruskalWallis non-parametric test of equality in median does not reject the null of no difference across all treatments $(\mathrm{p}$-value $=0.3973)$. Our subjects also display similar and not statistically different answers to the SOEP self-reported risk aversion measure.

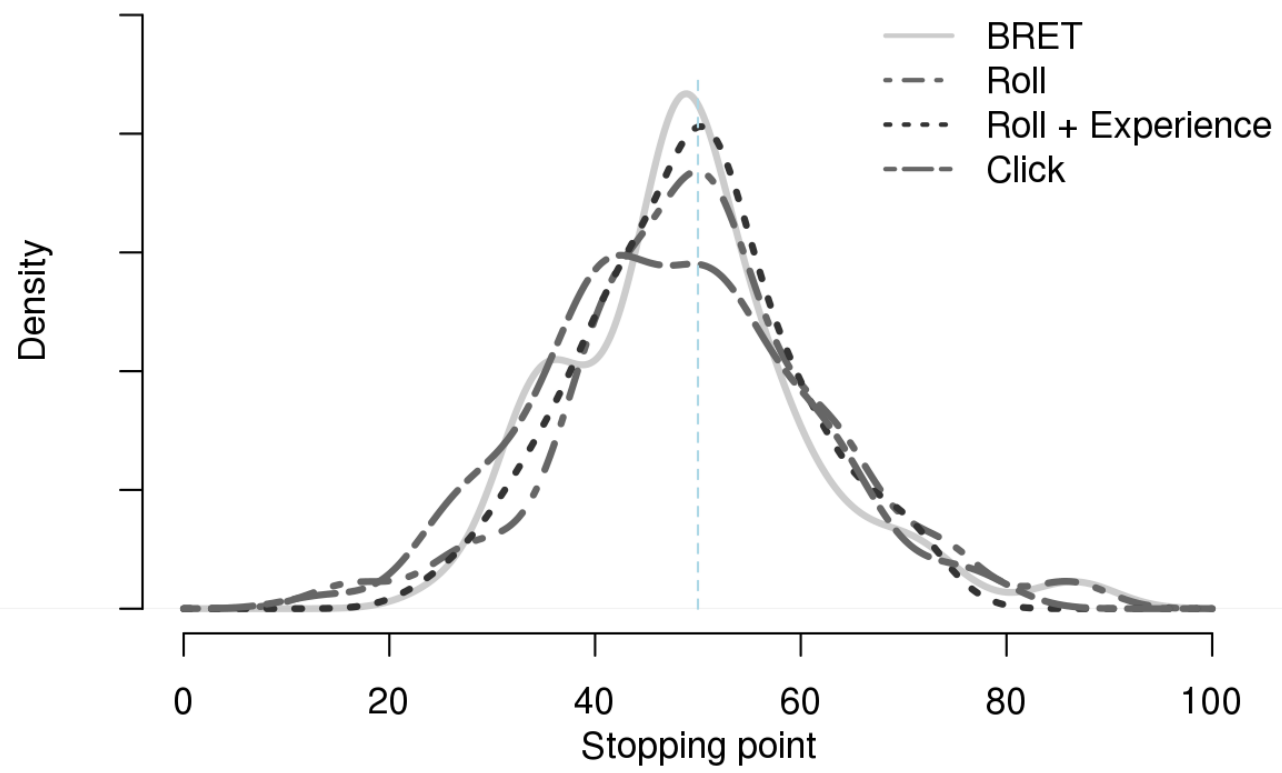

Figure 2: Kernel density estimate of choices, by treatment

Choices are also very similar in distribution. Figure 2 reports kernel density estimates of the number of boxes collected by treatment. The distribution of the baseline BRET and of the two Roll treatments is virtually identical. The Click treatment displays a flatter distribution, with less of a spike at 48-49 and some more weight on the tails. Still, equality between Click and Baseline distributions is not rejected (Mann-Whitney p-value $=0.4319$; KolmogorovSmirnov two-sample test, $\mathrm{p}$-value $=0.182$ ). Hence, our results strongly reject any effect of illusion of control when giving more active involvement both on the choice of the lottery and on the resolution of uncertainty. 
If illusion of control had a weak effect, the choice of the lottery is not likely to reflect it. Therefore, we also analyze whether illusion of control has at least a direct effect on the perceived likelihood of winning (variable belief), despite not being translated into a different choice. Note that in the BRET the objective probability of winning is by construction equal to $100-k$, where $k$ represents the number of boxes collected. Given that the probability of winning depends on the actual choice we also build the variable overconfidence, given by the difference between the perceived and objective probabilities of winning. ${ }^{8}$ Table 2 shows the average values of belief and overconfidence by treatment.

\begin{tabular}{lcccc}
\hline & \multicolumn{2}{c}{ Belief } & \multicolumn{2}{c}{ Overconfidence } \\
& mean & st.dev. & mean & st.dev. \\
\hline BRET & 51.84 & 15.83 & 0.99 & 17.76 \\
Roll & 50.57 & 15.40 & 0.84 & 17.56 \\
Roll Experience & 51.06 & 14.29 & 0.27 & 16.72 \\
Click & 51.13 & 15.25 & -1.73 & 19.86 \\
\hline
\end{tabular}

Table 2: Mean beliefs on probability of winning and overconfidence, by treatment

Both variables turn out to be statistically indistinguishable across conditions. The mean belief about the probability of winning does not differ (K-Wallis $\mathrm{p}$-value $=0.9881)$, and similar results are obtained adjusting for the objective probability of winning at the individual level $(\mathrm{K}-\mathrm{Wallis} \mathrm{p}$-value $=0.9718)$. Moreover, despite a considerable individual heterogeneity, the average overconfidence is very close to zero ( $p$-value $\geq 0.413$ in all treatments) meaning that subject hold correct beliefs on average. The distribution of overconfidence across treatments is also rather similar, as shown by the kernel density estimations of Figure 3. Therefore, also the elicitation of the perceived probability of winning does not detect any effect of illusion of control.

Finally, we report in Table 3 the results of a multivariate analysis aimed at investigating whether illusion of control appears in subgroups of our subjects pool and/or once we control for individual characteristics. Again, we do not find any significant effects of the experimental manipulations either on the choice (columns 1-2) or on the overconfidence of our subjects (columns 3-4). Males and females do not differ in terms of illusion of control, as emphasized by columns 2 and 4 which include an interaction between gender and our experimental conditions. ${ }^{9}$ A few control variables display a significant effect, although constant across all the experimental conditions. Subjects who are slower in completing the control questions are more underconfident, while the opposite behavioral trait characterizes those who make more mistakes, possibly signaling a lower understanding of the task. Finally, the number of siblings significantly correlate both with a more risk averse behavior and higher underconfidence.

\footnotetext{
${ }^{8}$ Note that the variable overconfidence operationalizes exactly the definition by Langer (1975) of illusion of control as an expectancy of a personal success probability inappropriately higher than the objective probability would warrant.

${ }^{9}$ Only in the Baseline females appear more risk averse, although at weak significance levels.
} 


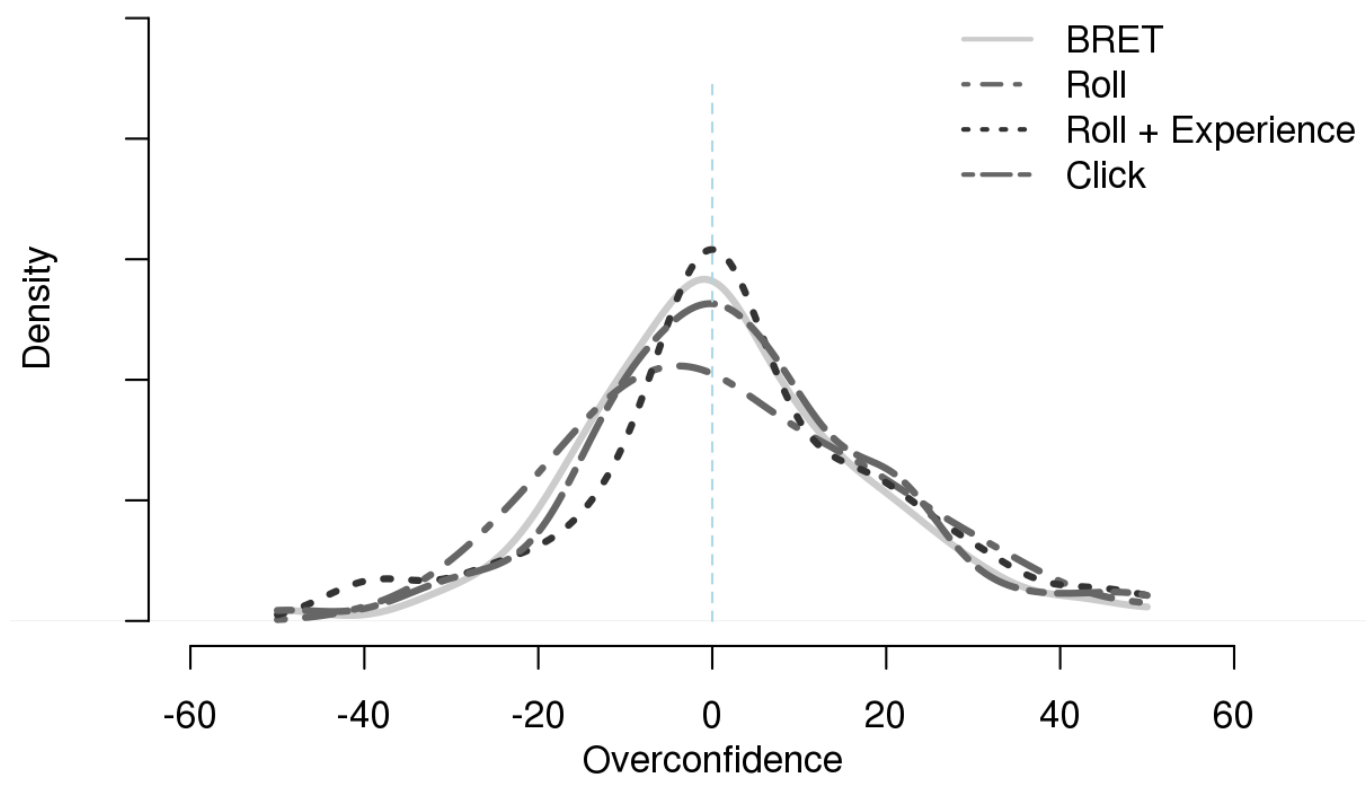

Figure 3: Kernel density estimate of overconfidence, by treatment

\section{Conclusion}

Illusion of control is the process by which people behave as if they have control in pure luck situations characterized by no objective control. Evidence of illusion of control is a recurrent finding in a large number of psychological studies, while the fewer laboratory experiments in economics find little to no effect. This striking difference in findings might be due to hypothetical bias: illusion of control might occur in hypothetical choices, typically used in psychological studies, but be crowded out by monetary incentives used in the lab by economists.

An alternative explanation could be the different domain of illusion of control that have been tested. The literature in experimental economics has been restricted to one type only of illusion of control, the one obtained increasing the involvement of the subjects in the resolution of uncertainty. This has usually been done by letting subjects roll the dice. By doing so illusion of control should bias upwards the perceived probability of being successful, and in turn affect the subject's choices in the task towards a more risk seeking behavior. A different source of illusion of control could stem from an enhanced involvement of the subject in the choice of the lottery to be played, i.e., in the decision under risk, rather than in the resolution of uncertainty. This second type of illusion of control has been tested in psychological studies only.

This paper aims at testing whether the dissonant results in the literature are due to the type of illusion of control that is triggered. We do so using the Bomb Risk Elicitation Task. The BRET allows us on the one hand to replicate the manipulations traditionally adopted in the economics literature, by letting the subjects roll the dice that determine the position of the bomb. On the other hand, it allows us to extend the investigation of illusion of control by varying the degree of control in the choice of the lottery to be played. We do so by allowing 


\begin{tabular}{|c|c|c|c|c|c|c|c|c|}
\hline \multirow[b]{2}{*}{ Click } & \multicolumn{2}{|c|}{$\begin{array}{c}(1) \\
\text { Choice }\end{array}$} & \multicolumn{2}{|c|}{$\begin{array}{c}(2) \\
\text { Choice }\end{array}$} & \multicolumn{2}{|c|}{$\begin{array}{c}(3) \\
\text { Overconfidence }\end{array}$} & \multicolumn{2}{|c|}{$\begin{array}{c}(4) \\
\text { Overconfidence }\end{array}$} \\
\hline & -1.896 & $(-1.03)$ & -2.644 & $(-0.93)$ & -2.443 & $(-0.89)$ & -1.919 & $(-0.45)$ \\
\hline Roll & 0.989 & $(0.53)$ & -3.232 & $(-1.13)$ & -0.375 & $(-0.14)$ & -4.900 & $(-1.15)$ \\
\hline Roll Experience & -0.376 & $(-0.17)$ & -2.903 & $(-0.89)$ & -0.434 & $(-0.13)$ & -4.785 & $(-0.99)$ \\
\hline Click $\times$ Female & & & -2.863 & $(-1.05)$ & & & -3.976 & $(-0.97)$ \\
\hline Roll $\times$ Female & & & 2.609 & $(0.99)$ & & & 3.965 & $(1.01)$ \\
\hline Roll Experience $\times$ Female & & & 0.297 & $(0.09)$ & & & 4.366 & $(0.91)$ \\
\hline Female & -1.177 & $(-0.81)$ & $-4.510^{*}$ & $(-1.69)$ & -0.100 & $(-0.05)$ & -3.606 & $(-0.91)$ \\
\hline Age & 0.232 & $(1.06)$ & 0.234 & $(1.07)$ & -0.145 & $(-0.45)$ & -0.154 & $(-0.47)$ \\
\hline Mistakes in $C Q$ & 0.037 & $(0.60)$ & 0.039 & $(0.62)$ & $0.160^{*}$ & $(1.73)$ & $0.157^{*}$ & $(1.69)$ \\
\hline Time in CQ/10 & -0.014 & $(-0.21)$ & -0.001 & $(-0.10)$ & $-0.216^{* *}$ & $(-2.09)$ & $-0.205^{* *}$ & $(-1.98)$ \\
\hline Nr. of siblings & $-0.938^{*}$ & $(-1.73)$ & $-0.996^{*}$ & $(-1.83)$ & $-1.600^{* *}$ & $(-1.98)$ & $-1.680^{* *}$ & $(-2.07)$ \\
\hline Constant & $45.60^{* * *}$ & $(8.44)$ & $47.41^{* * *}$ & $(8.67)$ & 10.50 & (1.30) & 12.61 & $(1.54)$ \\
\hline$N$ & \multicolumn{2}{|c|}{330} & \multicolumn{2}{|c|}{330} & \multicolumn{2}{|c|}{330} & \multicolumn{2}{|c|}{330} \\
\hline
\end{tabular}

Table 3: Multivariate regressions of choice and overconfidence

subjects to click to collect every single box vs. just choosing a stopping point.

Our results show no evidence of illusion of control across the board. Neither letting the subjects roll, nor letting them click to have a more active involvement in the choice of the lottery result in any effect either on the chosen number of boxes collected or on the perceived probability of avoiding the bomb. The experimental manipulations do not induce significant effects along a gender perspective, a results that survives the inclusion of individual-level controls in a multivariate analysis. The results of our experiments allows us to conclude that the striking difference of results across disciplines is not due to the fact that experimental economists have restricted their attention to one and not all possible forms of illusory control. Our results suggest that the dissonant results in the literature are likely due to incentives crowding out hypothetical biases. 


\section{References}

Benassi, V. A., Sweeney, P. D., Drevno, G. E., 1979. Mind over matter: Perceived success at psychokinesis. Journal of Personality and Social Psychology 37 (8), 1377-1386.

Charness, G., Gneezy, U., 01 2010. Portfolio Choice And Risk Attitudes: An Experiment. Economic Inquiry 48 (1), 133-146.

Chew, S. H., Sagi, J. S., 2008. Small worlds: Modeling attitudes toward sources of uncertainty. Journal of Economic Theory 139 (1), 1 - 24.

Crosetto, P., Filippin, A., August 2013. The 'bomb' risk elicitation task. Journal of Risk and Uncertainty $47(1), 31-65$.

Crosetto, P., Filippin, A., 2015. The sound of others: Surprising evidence of conformist behavior. Working Papers 2015-07, Grenoble Applied Economics Laboratory (GAEL).

Dohmen, T., Falk, A., Huffman, D., Sunde, U., Schupp, J., Wagner, G. G., 2011. Individual Risk Attitudes: Measurement, Determinants, And Behavioral Consequences. Journal of the European Economic Association 9 (3), 522-550.

Dunn, D. S., Wilson, T. D., 1990. When the Stakes are High: A Limit to the Illusion-of-Control Effect. Social Cognition 8 (3), 305-323.

Gino, F., Sharek, Z., Moore, D. A., 2011. Keeping the illusion of control under control: Ceilings, floors, and imperfect calibration. Organizational Behavior and Human Decision Processes 114 (2), 104 114.

Gneezy, U., Potters, J., 1997. An Experiment on Risk Taking and Evaluation Periods. The Quarterly Journal of Economics 112 (2), 631-45.

Langer, E. J., 1975. The illusion of control. Journal of Personality and Social Psychology 32 (2), 311328.

Li, K. K., 2011. Preference towards control in risk taking: Control, no control, or randomize? Journal of Risk and Uncertainty 43, 39-63.

Poon, D., 2011. Re-examining Behavioural Anomalies: Preference Reversal and Illusion of Control. Unpublished dissertation, School of Economics, The University of New South Wales.

Presson, P., Benassi, V., 1996. Illusion of control: A meta-analytic review. Journal of Social Behavior \& Personality.

Tversky, A., Wakker, P., 1995. Risk Attitudes and Decision Weights. Econometrica 63 (6), 1255-80.

van Rossum, G., 1995. Python reference manual. CWI Report CS-R9525.

Wagner, G. G., Frick, J. R., Schupp, J., 2007. The german socio-economic panel study (soep): Scope, evolution and enhancements. SOEPpapers on Multidisciplinary Panel Data Research 1, DIW Berlin, The German Socio-Economic Panel (SOEP). 


\section{Appendix A. Instructions}

Welcome screen

You are about to participate in an experiment in which following the instructions carefully, making good decisions, and with a bit of luck, you can earn money. Different participants may earn different amounts according to their choices. For your participation in the experiment you will earn an additional show-up fee of 2.5 Euro. All the monetary values during the experiment are expressed in Euro cents.

\section{Procedure Screen}

The experiment consists of 4 stages in the following order:

1. An Instruction Stage that we are currently going through. At the end of this stage you will be asked some control questions to verify your understanding of the task. After everybody answers correctly, we will proceed with the following stage.

2. A Decision Stage, in which you will make decisions and answer questions relevant to your final payoff.

3. A Questionnaire, in which you will be asked a few questions not related to your final payoff.

4. A Feedback Stage, in which your earnings from the experiment will be privately determined. You will not be given any feedback on the monetary outcome of your decisions before the Feedback Stage.

\section{Task instruction screen}

On your screen you will see a field composed of boxes numbered 1 through 100 . Your task is to decide on the number of boxes to collect out of 100 such boxes. You earn 10 Euro cents for each box collected. At any moment you can see the amount earned up to that point. Such earnings are only potential, however, because exactly one of these 100 boxes contains a time bomb, that if collected destroys all the boxes collected. You do not know the bombs location. You only know that it is equally likely to be in any of the 100 boxes. Moreover, even if you collect the time bomb, you will not know it until the end of the experiment.

[Baseline] To start collecting boxes you have to click the Play button that you will see on the screen. A box will automatically be collected at every second starting from the top left corner and following the numerical order until you decide to hit the Stop button when the number corresponds to how many boxes you want to collect. Once collected, each box disappears from the screen.

[Click] In order to collect each box you have to click once the Collect' button that you will see on the screen. Boxes are collected starting from the top left corner and following the numerical order until you decide to stop collecting and hit the Stop button when the number corresponds to how many boxes you want to collect. Once collected, each box disappears from the screen. At the end of the experiment, after answering some questions and filling out a short questionnaire, the number of the box containing the bomb will be randomly determined. 
[Baseline] An experimenter will come at your desk with two 10-sided dice. One will be used to determine the tens, the other the units. After letting you check that the dice are regular, the experimenter will roll them to determine the position of the bomb between 1 and 100 .

[Roll] An experimenter will come at your desk with two 10-sided dice. One will be used to determine the tens, the other the units. After letting you check that the dice are regular, you will roll them twice in order to familiarize with the procedure without this counting towards your final earnings. The third time you will roll them will determine the position of the bomb between 1 and 100 .

[Roll with Experience] Before the main task, an experimenter will distribute the two dice. You will have two minutes to familiarize with the dice and to check that the dice are regular. You can roll them as many times as you wish. At the end of the task an experimenter will come at your desk. You will then roll the dice once to determine the position of the bomb between 1 and 100.

If the number of the box in which the bomb is located is higher than the number of boxes you collected, you do not collect the bomb and you earn 10 Euro cents for each collected box. If the number of the box in which the bomb is located is lower than or equal to the number of boxes you collected, you do collect the bomb and you earn zero.

\section{Control Questions}

Please answer the following control questions. Answers to these questions are not relevant to your earnings. The computer will give you a feedback on whether your responses are correct or not. If you have any problems in answering, please raise your hand and an experimenter will come to assist you. After everyone answers correctly all the questions, we will proceed with the decision stage.

1. Suppose that the bomb is located in box 25 .

- If you collect 21 boxes, how much will you earn in Euro cent?

- If you collect 38 boxes, how much will you earn in Euro cent?

- If you collect 62 boxes, how much will you earn in Euro cent?

- If you collect 79 boxes, how much will you earn in Euro cent?

2. Now suppose that the bomb is located in box 75 .

- If you collect 21 boxes, how much will you earn in Euro cent?

- If you collect 38 boxes, how much will you earn in Euro cent?

- If you collect 62 boxes, how much will you earn in Euro cent?

- If you collect 79 boxes, how much will you earn in Euro cent?

3. What is the probability of the bomb being in boxes number:

- 51 through 70

- 21 though 50

- 1 through 10 
- 61 through 100

4. The location of the bomb depends on how many boxes you decide to collect. [YES/NO]

5. Suppose the location of the bomb is identical to the number of boxes you collect. Will you earn a positive amount? [YES/NO]

6. Who will roll the dice to determine the position of the bomb? [Me/Experimenter/Another participant]

Please note that the numbers used in these questions are for illustrative purposes only. They are not meant to be a guidance for your choice.

Earmuffs screen

Now if everything is clear please wear the earmuffs you see on your desk. You will be notified in another screen later when you have to remove them.

\section{Decision screen}

Please choose the number of boxes to collect.

[Baseline] Hit the Play button to start collecting the boxes. One box will be automatically collected at every second.

[Click] In order to collect each box you have to click once the Collect' button.

Boxes are collected starting from the top left corner and following the numerical order. When the number corresponds to how many boxes you want to collect hit the Stop button. Then confirm your choice by clicking on Confirm. 